\title{
Coherent measures of risk from a general equilibrium perspective
}

Citation for published version (APA):

Csóka, P., Herings, P. J. J., \& Kóczy, L. Á. (2007). Coherent measures of risk from a general equilibrium perspective. Journal of Banking \& Finance, 31, 2517-2534. https://doi.org/10.1016/j.jbankfin.2006.10.026

Document status and date:

Published: 01/01/2007

DOI:

10.1016/j.jbankfin.2006.10.026

Document Version:

Publisher's PDF, also known as Version of record

Document license:

Taverne

Please check the document version of this publication:

- A submitted manuscript is the version of the article upon submission and before peer-review. There can be important differences between the submitted version and the official published version of record.

People interested in the research are advised to contact the author for the final version of the publication, or visit the DOI to the publisher's website.

- The final author version and the galley proof are versions of the publication after peer review.

- The final published version features the final layout of the paper including the volume, issue and page numbers.

Link to publication

\footnotetext{
General rights rights.

- You may freely distribute the URL identifying the publication in the public portal. please follow below link for the End User Agreement:

www.umlib.nl/taverne-license

Take down policy

If you believe that this document breaches copyright please contact us at:

repository@maastrichtuniversity.nl

providing details and we will investigate your claim.
}

Copyright and moral rights for the publications made accessible in the public portal are retained by the authors and/or other copyright owners and it is a condition of accessing publications that users recognise and abide by the legal requirements associated with these

- Users may download and print one copy of any publication from the public portal for the purpose of private study or research.

- You may not further distribute the material or use it for any profit-making activity or commercial gain

If the publication is distributed under the terms of Article $25 \mathrm{fa}$ of the Dutch Copyright Act, indicated by the "Taverne" license above, 


\title{
Coherent measures of risk from a general equilibrium perspective is
}

\author{
Péter Csóka, P. Jean-Jacques Herings ${ }^{*, 1}$, László Á. Kóczy ${ }^{2}$ \\ Department of Economics, Universiteit Maastricht, P.O. Box 616, 6200 MD, Maastricht, The Netherlands
}

Received 24 April 2006; accepted 24 October 2006

Available online 24 January 2007

\begin{abstract}
Coherent measures of risk defined by the axioms of monotonicity, subadditivity, positive homogeneity, and translation invariance are recent tools in risk management to assess the amount of risk agents are exposed to. If they also satisfy law invariance and comonotonic additivity, then we get a subclass of them: spectral measures of risk. Expected shortfall is a well-known spectral measure of risk.

We investigate the above mentioned six axioms using tools from general equilibrium (GE) theory. Coherent and spectral measures of risk are compared to the natural measure of risk derived from an exchange economy model, which we call the GE measure of risk. We prove that GE measures of risk are coherent measures of risk. We also show that spectral measures of risk are GE measures of risk only under stringent conditions, since spectral measures of risk do not take the regulated entity's relation to the market portfolio into account. To give more insights, we characterize the set of GE measures of risk via the pricing kernel property.

(C) 2007 Elsevier B.V. All rights reserved.
\end{abstract}

\footnotetext{
is We are grateful to two anonymous referees and conference and seminar participants in Maastricht, Amsterdam, Budapest and Lisbon for helpful comments.

* Corresponding author. Tel.: +31 43388 3824; fax: +31 433884878.

E-mail addresses: P.Csoka@algec.unimaas.nl (P. Csóka), P.Herings@algec.unimaas.nl (P.J.-J. Herings), L.Koczy@algec.unimaas.nl (L.Á. Kóczy).

${ }^{1}$ The author would like to thank the Netherlands Organisation for Scientific Research (NWO) for financial support.

${ }^{2}$ The author would like to thank funding by the Netherlands Organisation for Scientific Research (NWO) and by the European Union under the Marie Curie Intra-European Fellowship MEIF-CT-2004-011537.
} 
JEL classification: D51; G10; G12

Keywords: Coherent measures of risk; General equilibrium theory; Exchange economies; Asset pricing

\section{Introduction}

Risk management is of crucial importance considering the enormous financial risk our economy is exposed to. The risks of many economic agents are regulated by various institutions. For example, if a financial trader wants to sell options, which give the buyer rights of buying or selling at a given price during a specified time horizon (or at a given time), he has to fulfil margin requirements, i.e. he has to deposit some cash or some other riskless and liquid instrument. An exchange's clearing firm, which is responsible for the promises to all parties of transactions being securely completed, requires margin deposits. A measure of risk can be used to determine the margin requirement. The riskier the trader's portfolio, the more the margin requirement should be.

Other external regulators, at an international level, are the International Actuarial Association (IAA) and the International Accounting Standards Board (IASB), who determine the capital requirements for insurance companies. Similarly, the Basel Committee gives guidelines for the acceptable level of capital on banking supervision. Since a government or central bank could be a lender of last resort for these institutions, and the default of them could cause serious problems, they are regulated as well. As an internal regulator, a portfolio manager has to regulate the risk of its traders. In the context of a multi-division firm setting, the head-office may also set risk-limits for the divisions. Internally the risk values can also be used for planning and performance evaluation. It is therefore crucial to measure risk in an appropriate way.

We will use the term portfolio when referring to a risky entity (portfolio, firm, insurance company, bank, etc.). The value of a portfolio might change due to all kinds of uncertain events. We relate risk to the probability distribution of the future value of the portfolio. For the sake of simplicity in this paper we use discrete random variables. Our approach can be extended to the case of continuous risks and risks with unbounded support. All this requires is an analysis of competitive equilibrium in such an environment. The interested reader is referred to Chapter 10 of Duffie (2001).

A measure of risk assigns a real number to a random variable. It is the minimal amount of cash the regulated agent has to add to his portfolio, and to invest in a zero coupon bond. Coherent measures of risk (Artzner et al., 1999) are defined by four axioms: monotonicity, subadditivity, positive homogeneity and translation invariance. When adding two more axioms: law invariance and comonotonic additivity we get a subclass of coherent measures of risk, namely spectral measures of risk (Acerbi, 2002). Expected shortfall is a well-known spectral measure of risk (Acerbi and Tasche, 2002). For an introduction to risk measures and the above mentioned axioms see for instance Chapter 4 of Föllmer and Schied (2002).

Our approach is to model the situation at hand as an exchange economy in a general equilibrium (GE) setting, and determine which axioms are compatible with this model, and whether other axioms emerge as natural. This approach has the advantage that it recognizes the fact that the risk of a portfolio depends on the other assets present in the economy (the market portfolio), an insight that is generated immediately by the Capital Asset 
Pricing Model as developed by Sharpe (1964) and Lintner (1965). By doing so we would like to contribute to the research agenda that connects finance to GE theory, see for instance Geanakoplos and Shubik (1990), Magill and Quinzii (1996), Leroy and Werner (2001), or Jaschke and Küchler (2001). The corresponding measure of risk of a portfolio would be the amount of cash needed to sell the risk involved in the portfolio to the market. More precisely, the so-called GE measure of risk of a portfolio would be the negative of its equilibrium market price.

We prove that GE measures of risk are coherent and comonotonic additive measures of risk. However, GE measures of risk fail to satisfy law invariance, i.e. they are functions of not only the probability distributions of the portfolios, since they also take the regulated entity's relation to the market portfolio into account. Nevertheless we show that GE measures of risk satisfy a generalized notion of law invariance. To check on which domain spectral measures of risk are GE measures of risk, we consider a general domain for the measures of risk. We find that the corresponding domain is very small. To give more insights, we characterize GE measures of risk as the only measures of risk satisfying the property that we call the pricing kernel property.

The structure of the paper is as follows. In Section 2 we discuss coherent measures of risk. In Section 3 spectral measures of risk are considered. Using the exchange economy model of Section 4 the properties of GE measures of risk are investigated in Section 5. In Section 6 we show that spectral measures of risk are GE measures of risk only under stringent conditions, and we characterize GE measures of risk via the pricing kernel property. We conclude in Section 7.

\section{Coherent measures of risk}

Consider a set $V \subseteq \mathbb{R}^{S}$ of realization vectors, where $S$ denotes the number of states of nature. State of nature $s$ occurs with probability $p_{s}>0$ and $\sum_{s=1}^{S} p_{s}=1$. The vector $X \in V$ represents a portfolio's (firm's, insurance company's, bank's, etc.) possible profit and loss realizations on a common chosen future time horizon, say at $t=1$. The amount $X_{s}$ is the portfolio's payoff in state of nature $s$. Negative values of $X_{s}$ correspond to losses. The inequality $Y \geqslant X$ means that $Y_{s} \geqslant X_{s}$ for all $s=1, \ldots, S$. We define $R_{+}=[0, \infty), R_{++}=$ $(0, \infty), R_{-}=(-\infty, 0]$, respectively. The discrete random variable generated by $p \in \mathbb{R}_{++}^{S}$ and $X \in V$ is denoted by $\widehat{X}$, i.e. $P\left(\widehat{X}=X_{s}\right)=p_{s}$, for all $s=1, \ldots, S$.

A measure of risk is a function $\rho: V \rightarrow \mathbb{R}$ measuring the risk of a portfolio from the perspective of the present $(t=0)$. It is the minimal amount of cash the regulated agent has to add to his portfolio, and to invest in a reference instrument today, such that it ensures that the risk involved in the portfolio is acceptable to the regulator. ${ }^{3}$ We assume that the reference instrument has payoff 1 in each state of nature at $t=1$, thus its realization vector is $\mathbf{1}=(1, \ldots, 1) \in V$. The reference instrument is riskless in the "classical sense", having no uncertainty in its payoffs. It is most natural to think of it as a zero coupon bond. The price of the reference instrument, the discount factor is denoted by $\delta \in \mathbb{R}_{+}$.

We adjust the definition of coherent measures of risk to the discrete case with realization vectors on a general domain $V$ as follows.

\footnotetext{
${ }^{3}$ The measure of risk can also be negative, meaning that a portfolio remains acceptable if a certain amount of cash is withdrawn from it.
} 
Definition 2.1. A function $\rho: V \rightarrow \mathbb{R}$ is called a coherent measure of risk (Artzner et al., 1999) if it satisfies the following axioms:.

1. Monotonicity: for all $X, Y \in V$ such that $Y \geqslant X$, we have $\rho(Y) \leqslant \rho(X)$.

2. Subadditivity: for all $X, Y \in V$ such that $X+Y \in V$, we have $\rho(X+Y) \leqslant \rho(X)+\rho(Y)$.

3. Positive homogeneity: for all $X \in V, h \in \mathbb{R}_{+}$such that $h X \in V$, we have $\rho(h X)=h \rho(X)$.

4. Translation invariance: for all $X \in V$ and $a \in \mathbb{R}$ such that $X+a \mathbf{1} \in V$, we have $\rho(X+a \mathbf{1})=\rho(X)-\delta a$.

The axioms are motivated as follows.

By monotonicity, if a portfolio $Y$ is always worth at least as much as $X$ (event by event), then $Y$ cannot be riskier than $X$.

Subadditivity says that if we combine two portfolios, the risk is not greater than the sum of the risks associated with each: it captures the notion of diversification. If an exchange's measure of risk would fail to satisfy this property, then a trader could be better off by splitting his position, opening two accounts and decreasing the margin requirements. The same motivation applies to firms, banks, insurance companies, etc. For instance, for the case of internal capital budgeting, if the measure of risk satisfies subadditivity, then the head office can be sure that by setting risk limits to the divisions, the risk of the whole firm will be smaller than the sum of the individual risk limits. This enables the head office to decentralize the risk constraints.

Positive homogeneity requires that portfolio size should linearly influence risk.

Translation invariance ensures that the measure of risk is expressed in appropriate units. It means that investing an amount $\delta a>0$ (or $\delta a<0)$ of cash into $a$ units of reference instrument at $t=0$ leads to the payoff vector $a \mathbf{1}$ at $t=1$ and decreases (respectively: increases) the measure of risk by $\delta a$.

For further motivation of the axioms see Artzner et al. (1999).

The four axioms above seem natural, but one can easily create counterexamples showing that the rather popular Value at Risk (VaR) is not subadditive in general and hence it is not a coherent measure of risk, see for instance Artzner et al. (1999), or Embrechts et al. (2002). However, according to Embrechts et al. (2002) VaR is coherent for elliptically distributed risks. Moreover, Ibragimov (2005) shows that VaR satisfies the coherency axioms for not extremely heavy-tailed risks with finite means and convolutions of $\alpha$-symmetric risks with $\alpha>1$, but VaR always lacks the coherency property for extremely heavy-tailed risks with infinite first moments and convolutions of $\alpha$-symmetric risks with $\alpha<1$. As a byproduct checking the relevance of the coherency axioms from a general equilibrium perspective enables us to assess this criticism on VaR as well.

\section{Spectral measures of risk}

Acerbi (2002) treats spectral measures of risk in case of discrete random variables with equiprobable outcomes, i.e. when $p_{1}=\cdots=p_{S}=1 / S$, as a special case. He assumes that the discount factor is 1 , and the domain is $\mathbb{R}^{S}$. In this paper we explicitly want to use the discount factor, which for the moment is exogenously determined here as the price of the reference instrument. Since in the general equilibrium model the discount factor will be determined endogenously, we have to multiply Acerbi (2002)'s definition by $\delta$. Our model is a generalization also in the sense that we consider a general domain 
$V \subseteq \mathbb{R}^{S}$. The definition of spectral measures of risk with equiprobable outcomes is as follows.

Let us introduce the ordered statistics $X_{S: S}$ given by the ordered values of the $S$-tuple $X_{1}, \ldots, X_{S}$, i.e. $\left\{X_{1: S}, \ldots, X_{S: S}\right\}=\left\{X_{1}, \ldots, X_{S}\right\}$ and $X_{1: S} \leqslant X_{2: S} \leqslant \cdots \leqslant X_{S: S}$.

Definition 3.1. Let the outcomes be equiprobable. Consider a domain $V \subseteq \mathbb{R}^{S}$, and a vector $\phi \in \mathbb{R}^{S}$. The measure $M_{\phi}: V \rightarrow \mathbb{R}$ defined by

$$
M_{\phi}(X)=-\delta \sum_{s=1}^{S} \phi_{s} X_{s: S}
$$

is a spectral measure of risk if $\phi \in \mathbb{R}^{S}$ satisfies the conditions

1. Nonnegativity: $\phi_{s} \geqslant 0$ for all $s=1, \ldots, S$,

2. Normalization: $\sum_{s=1}^{S} \phi_{s}=1$,

3. Monotonicity: $\phi_{s}$ is non-increasing, i.e. $\phi_{s_{1}} \geqslant \phi_{s_{2}}$ if $s_{1}<s_{2}$ and $s_{1}, s_{2} \in\{1, \ldots, S\}$.

Spectral measures of risk are calculated as discounted weighted average losses, with non-increasing weights, with the highest weight on the worst outcome. The weight vector $\phi$ is the so-called risk spectrum. It can be interpreted as expressing the attitude toward risk. As a special case we have the following definition.

Definition 3.2. Let $k \in\{1, \ldots, S\}$. The k-expected shortfall of the realization vector $X$ is defined by

$$
\operatorname{ES}_{k}(X)=-\delta \sum_{s=1}^{k} \frac{1}{k} X_{s: S} .
$$

The $k$-expected shortfall is the discounted average of the worst $k$ outcomes. With an unrestricted domain, i.e. if $V=\mathbb{R}^{S}$, Acerbi (2002) has the following result.

Proposition 3.3. Assume $V=\mathbb{R}^{S}$. A measure of risk $M_{\phi}: V \rightarrow \mathbb{R}$ is coherent if and only if it is a spectral measure of risk as defined by Definition 3.1 .

Proof. Acerbi (2002, Theorem 5.3).

Of course on a restricted domain, i.e. if $V \subset \mathbb{R}^{S}$, spectral measures of risk still satisfy the four coherency axioms. However, as the following three examples show, depending on $V$ one might find other measures of risk $M_{\phi}: V \rightarrow \mathbb{R}$ that are coherent but not spectral measures of risk.

Example 3.4. As a trivial example, let $V$ contain only one vector, $X$. In this case all measures of risk, i.e. all functions $\rho: V \rightarrow \mathbb{R}$ satisfy the six axioms, since there are no two vectors on which the axioms of monotonicity, subadditivity, etc. could be checked. Note that in this case spectral measures of risk are those functions $\rho: V \rightarrow \mathbb{R}$ that satisfy

$$
-\frac{\delta}{S} \sum_{s=1}^{S} X_{s: S} \leqslant \rho(X) \leqslant-\delta X_{1: S} .
$$


For all functions $\rho$ satisfying (3) there is $\phi \in \mathbb{R}^{S}$ such that $M_{\phi}=\rho$ and $M_{\phi}$ is a spectral measure of risk.

Interestingly, Eq. (3) can also be justified using majorization theory. A vector $\phi \in \mathbb{R}^{S}$ is said to be majorized by a vector $\gamma \in \mathbb{R}^{S}$, written $\phi \prec \gamma$, if $\sum_{i=k}^{S} \phi_{i: S} \leqslant \sum_{i=k}^{S} \gamma_{i: S}, k=2, \ldots, S$ and $\sum_{i=1}^{S} \phi_{i: S}=\sum_{i=1}^{S} \gamma_{i: S}$. It is easy to see that for a risk spectrum $\phi \in \mathbb{R}^{S}$ we have that

$$
\left(\frac{1}{S}, \ldots, \frac{1}{S}\right) \prec\left(\phi_{1}, \ldots, \phi_{S}\right) \prec(1,0, \ldots, 0) .
$$

It follows from 3.H.2.c and 4.b.7 in Marshall and Olkin (1979) that $M_{\phi}$ is Schur-convex in $\phi$, i.e. if $\phi_{1} \prec \phi_{2}$, then for any $X \in V$ we have that $M_{\phi_{1}}(X) \leqslant M_{\phi_{2}}(X)$. From this observation and the relations in (4), Eq. (3) follows immediately.

Example 3.5. In his proof of Theorem 5.3 (Proposition 3.3 here) Acerbi (2002) shows for the case $V=\mathbb{R}^{S}$ that if a measure of risk $M_{\phi}: V \rightarrow \mathbb{R}$ is monotone, then its risk spectrum $\phi$ should be nonnegative. If for a certain $s$ we have that $\phi_{s}<0$, then for any $X \in V$ an increase in $X_{s: S}$ gives rise to a higher measure of risk, contradicting monotonicity.

However, for many domains $V$, positivity is not required for monotonicity. Consider a domain $V=\mathbb{R}_{-}^{S-1} \times\{0\}$. In this case the best outcome of any portfolio is zero, thus the weight of the best outcome, $\phi_{S}$ can be negative, since the best outcome cannot be increased. So although $\phi$ is not nonnegative everywhere, the measure of risk remains monotone.

Example 3.6. In his proof of Theorem 5.3 (Proposition 3.3 here) Acerbi (2002) also shows for the case $V=\mathbb{R}^{S}$ that if a measure of risk $M_{\phi}: V \rightarrow \mathbb{R}$ is translation invariant, then its risk spectrum $\phi$ should be normalized. If $\phi$ is not normalized, then it is easy to check that translation invariance fails. It suffices to consider $M_{\phi}(X)$ and $M_{\phi}(Y)$, where $Y=X+a \mathbf{1}$, and $a \in \mathbb{R}, a \neq 0$.

For general domains $V$, normalization is not required for translation invariance. For instance, translation invariance is trivially satisfied if there are no two vectors $X$ and $Y$ such that $X=Y+a \mathbf{1}$ for some $a \in \mathbb{R}, a \neq 0$. In this case translation invariance does not have bite and normalization can be omitted.

Any measure of risk $M_{\phi}: V \rightarrow \mathbb{R}$ and in particular spectral measures of risk can be shown to satisfy the axioms of law-invariance and comonotonic additivity, since they use the ordered statistics of a portfolio. The definitions of these two axioms are as follows.

Definition 3.7. A measure of risk $\rho: V \rightarrow \mathbb{R}$ is law invariant if for all $X, Y \in V$ such that for all $s=1, \ldots, S$,

$$
\operatorname{Pr}\left(\widehat{X}=X_{s}\right)=\operatorname{Pr}\left(\widehat{Y}=X_{s}\right),
$$

we have $\rho(X)=\rho(Y)$.

Law invariance of $\rho$ means that the measure of risk is a function of the probability distribution (law) only. Note that two random variables $\widehat{X}$ and $\widehat{Y}$ can be different despite the fact that they have the same probability distribution. Acerbi (2004) explains that law 
invariance can be thought of as the property of "being estimable from empirical data". We will point out in Section 5 that the portfolios' relations to the economy as a whole are also important, which will be shown to violate the law invariance axiom. For instance, if two portfolios have different covariances with the market portfolio, then the regulator may perceive their risk differently, even if their probability distribution is the same.

Another characteristic of spectral measures of risk is comonotonic additivity.

Definition 3.8. Two realization vectors $X \in \mathbb{R}^{S}$ and $Y \in \mathbb{R}^{S}$ are comonotonic if for all $s_{1}, s_{2} \in\{1, \ldots, S\}$

$$
\left(X_{s_{1}}-X_{s_{2}}\right)\left(Y_{s_{1}}-Y_{s_{2}}\right) \geqslant 0 \text {. }
$$

A map $\rho: V \rightarrow \mathbb{R}$ is comonotonic additive if given two comonotonic realization vectors $X, Y \in V$ such that $X+Y \in V$ the map displays additivity, i.e. we have $\rho(X+Y)=\rho(X)+$ $\rho(Y)$.

Comonotonicity is a very strong form of dependence that two realization vectors $X$ and $Y$ can display. If two portfolios are comonotonic, then their value will always move up and down together event by event, providing no diversification at all when added to each other. The measure of risk of a portfolio consisting of two comonotonic subportfolios should therefore be equal to the sum of the measures of risk of the subportfolios. Using random variables the definition of comonotonicity is as follows.

Definition 3.9. Two random variables $\widehat{X}$ and $\widehat{Y}$ are comonotonic if $\widehat{X}=f(\widehat{Y})$ (almost surely) for some increasing function $f: \mathbb{R} \rightarrow \mathbb{R}$.

The definitions of comonotonicity for realization vectors and random variables are equivalent.

For two random variables with continuous distributions $\widehat{X}$ and $\widehat{Y}$ the inequality in (5) holds for their realization vectors $X$ and $Y$ if and only if we have that the probability of concordance defined by $P\left(\left(\widehat{X}-\widehat{X}^{\prime}\right)\left(\widehat{Y}-\widehat{Y}^{\prime}\right) \geqslant 0\right)=1$ and consequently the probability of discordance given by $P\left(\left(\widehat{X}-\widehat{X}^{\prime}\right)\left(\widehat{Y}-\widehat{Y}^{\prime}\right) \leqslant 0\right)=0$, where $\widehat{X}^{\prime}$ and $\widehat{Y}^{\prime}$ are independent copies of $\widehat{X}$ and $\widehat{Y}$. In this case, one of the commonly used measures of dependence, Kendall's tau defined by

$$
\rho_{\tau}=P\left(\left(\widehat{X}-\widehat{X}^{\prime}\right)\left(\widehat{Y}-\widehat{Y}^{\prime}\right) \geqslant 0\right)-P\left(\left(\widehat{X}-\widehat{X}^{\prime}\right)\left(\widehat{Y}-\widehat{Y}^{\prime}\right) \leqslant 0\right)
$$

reaches its maximum value, i.e. $\rho_{\tau}=1$. From Theorem 3 in Embrechts et al. (2002) it follows that $\rho_{\tau}=1$ is equivalent with saying that $\widehat{X}$ and $\widehat{Y}$ are comonotonic as defined by Definition 3.9. For Kendall's tau and related measures of dependence see for instance Chapter 5 in Nelsen (1999).

Tasche (2002) shows that in the continuous, non-atomic case spectral measures of risk are the only coherent measures of risk satisfying law-invariance and comonotonic additivity. As his proof requires non-atomic probability distributions, in the discrete, equiprobable case a new proof is required for the analogous statement. The proposition requires an unrestricted domain, i.e. $V=\mathbb{R}^{S}$.

Proposition 3.10. Assume that the outcomes are equiprobable and $V=\mathbb{R}^{S}$. Then a measure of risk is coherent, law invariant and comonotonic additive if and only if it is a spectral measure of risk as defined by Definition 3.1. 


\section{Proof}

$(\Leftarrow)$ The coherency part follows from Proposition 3.3. Law invariance and comonotonic additivity follows from the fact that spectral measures of risk are using the ordered statistics of a realization vector.

$(\Rightarrow)$ Take any law invariant and comonotonic additive measure of risk $\rho: \mathbb{R}^{S} \rightarrow \mathbb{R}$ satisfying the four coherency axioms. We will show that law invariance and comonotonic additivity of $\rho$ implies that it can be written in the form of Eq. (1), i.e. there exists a vector $\phi \in \mathbb{R}^{S}$ such that for all $X \in \mathbb{R}^{S}$ we have that

$$
\rho(X)=M_{\phi}(X)=-\delta \sum_{s=1}^{S} \phi_{s} X_{s: S}
$$

Law invariance of $\rho$ implies that for all $X \in \mathbb{R}^{S}$ any permutation of $X$ has the same measure of risk. A particular permutation is the ordered statistics vector of $X,\left[X_{s: S}\right]$. Using the notation $\bar{X}=\left\{X \in \mathbb{R}^{S} \mid X_{1} \leqslant X_{2} \leqslant \cdots \leqslant X_{S}\right\}$ for the space of ordered statistics, we have that for all $X \in \mathbb{R}^{S}$ the measure of risk $\rho$ can be written as a function $f: \bar{X} \rightarrow \mathbb{R}$ of the ordered statistics vector,

$$
\rho(X)=f\left(\left[X_{s: S}\right]\right) \text {. }
$$

Comonotonic additivity of $\rho$ implies that $f$ is linear on $\bar{X}$. Choosing $S$ independent vectors in $\bar{X}$ implies that $f$ is additive separable with constant weight functions $\bar{\phi}_{1}, \ldots, \bar{\phi}_{S} \in \mathbb{R}$, i.e.

$$
f\left(\left[X_{s: S}\right]\right)=\bar{\phi}_{1} X_{1: S}+\cdots+\bar{\phi}_{S} X_{S: S} \text {. }
$$

Using $\phi_{s}=-\frac{\bar{\phi}_{s}}{\delta}, s=1, \ldots, S$ we have that Eq. (7) is satisfied. Proposition 3.3 implies that $\rho$ should be a spectral measure of risk.

It is cumbersome to generalize the definition of spectral measures of risk to the case in which the outcomes are not equiprobable. Consider the following example.

Example 3.11. In Table 1 we have four states of nature with equal probability of occurrence. Portfolios $X$ and $Y$, their ordered statistics, and the risk spectrum $\phi$ are also given. A straightforward calculation gives $M_{\phi}(X)=0$, and $M_{\phi}(Y)=3.5 \delta$.

Since the portfolios $X$ and $Y$ have the same outcome in states of nature 2, 3 and 4, we can merge those states. This operation leads to Table 2, with portfolios $X^{\prime}$ and $Y^{\prime}$, where the generalized discrete risk spectrum $\phi^{g}$ is given by $\phi_{1}^{g}=\phi_{1}$ and $\phi_{2}^{g}=\phi_{2}+\phi_{3}+\phi_{4}$.

Table 1

Spectral measures of risk with equiprobable outcomes

\begin{tabular}{lllllll}
\hline$s$ & $p_{s}$ & $X_{s}$ & $X_{s: S}$ & $Y_{s}$ & $Y_{s: S}$ & $\phi_{s}$ \\
\hline 1 & 0.25 & -3 & -3 & 1 & -4 & 0.4 \\
2 & 0.25 & 2 & 2 & -4 & -4 & 0.3 \\
3 & 0.25 & 2 & 2 & -4 & -4 & 0.2 \\
4 & 0.25 & 2 & 2 & -4 & 1 & 0.1 \\
\hline
\end{tabular}

Table 2

Spectral measures of risk with not equiprobable outcomes

\begin{tabular}{lllllll}
\hline$s$ & $p_{s}^{\prime}$ & $X_{s}^{\prime}$ & $X_{s: S}^{\prime}$ & $Y_{s}^{\prime}$ & $Y_{s: S}^{\prime}$ & $\phi_{s}^{g}$ \\
\hline 1 & 0.25 & -3 & -3 & 1 & -4 & 0.4 \\
2 & 0.75 & 2 & 2 & -4 & 1 & 0.6 \\
\hline
\end{tabular}


By defining $M_{\phi^{g}}(X)=-\delta \sum_{s=1}^{S} \phi_{S}^{g} X_{s: S}$ we have that $M_{\phi}(X)=M_{\phi^{g}}\left(X^{\prime}\right)=0$, but $M_{\phi}(Y)=3.5 \delta$, whereas $M_{\phi^{g}}\left(Y^{\prime}\right)=\delta$. To avoid this problem, within the discrete setting a different $\phi^{g}$ should be specified for all the orderings of the portfolio vectors. This would rather complicate the analysis and would not add much value since with splitting up the states and increasing their number any discrete distribution can be arbitrarily closely approximated by equiprobable states. Thus we will use the discrete, equiprobable version of spectral measures of risk as defined in Definition 3.1 in the sequel.

\section{An exchange economy model}

Next we discuss the four axioms underlying coherent measures of risk and the additional two axioms of spectral measures of risk from a general equilibrium perspective. We do this by checking the validity of the six axioms on the natural measure of risk derived from an exchange economy model, which we call the General Equilibrium (GE) measure of risk. The GE measure of risk of a portfolio is the amount of cash needed to sell the risk involved in the portfolio to the market. More precisely, it is the minimal amount of cash needed to add to the portfolio, such that its market price becomes nonnegative. It is easy to see that the GE measure of risk of a portfolio is the negative of its equilibrium market price.

To specify the GE measure of risk we consider an exchange economy model with two time periods $(t=0, t=1)$ and uncertainty concerning the state of nature in period $t=1$. We assume that for each state $s$ in the set $\{1, \ldots, S\}$ its probability of occurrence is objectively known, $p_{s}=1 / S .{ }^{4}$ Period $t=0$ is identified with state $s=0$. There is a unique nondurable commodity (income) in each state of nature $s=0, \ldots, S$.

The portfolios (firms, insurance companies, banks, etc.) are represented as exogenously given realization vectors in $\mathbb{R}^{S}$. Their payoff is a profit or loss in state of nature $s=1, \ldots, S$.

To simplify the analysis, we assume that the economy can be modeled by means of a representative agent. However, it is not difficult to extend the model to the case with heterogeneous agents. We assume that the consumption set of the agent is $\mathbb{R}$ in all states of nature. His consumption stream is denoted by $c=\left(c_{0}, \ldots, c_{S}\right) \in \mathbb{R}^{S+1}$.

The agent's preferences are represented by a von Neumann-Morgenstern utility function $u: \mathbb{R}^{S+1} \rightarrow \mathbb{R}$, given by

$$
u\left(c_{0}, \ldots, c_{S}\right)=v_{0}\left(c_{0}\right)+\bar{\delta} \sum_{s=1}^{S} p_{s} v\left(c_{s}\right),
$$

where $v_{0}: \mathbb{R} \rightarrow \mathbb{R}$ and $v: \mathbb{R} \rightarrow \mathbb{R}$ are elementary (Bernoulli) utility functions in state 0 and states $s=1, \ldots, S$, respectively; the scalar $\bar{\delta} \in \mathbb{R}_{++}$is the subjective discount factor of the agent.

Moreover, the representative agent is characterized by his (aggregate) endowment $\omega=\left(\omega_{0}, \ldots, \omega_{S}\right) \in \mathbb{R}^{S+1}$. The endowment expresses the agent's consumption possibilities without trade, depending on the realization of the state of nature. To distinguish between the sure zero-period endowment and the random first-period endowment, we define $\tilde{\omega}=\left(\omega_{1}, \ldots, \omega_{S}\right)$.

\footnotetext{
${ }^{4}$ We shall only employ the equiprobable assumption when comparing GE measures of risk to spectral measures of risk.
} 
We assume that income transfers across all states are possible, i.e. markets are complete. Without loss of generality we assume that there are $S$ contingent contracts.

Definition 4.1. A contingent contract for state $s(s=1, \ldots, S)$ is a promise to deliver one unit of income in state $s$ and nothing otherwise. The price of the contingent contract, the state price, expressed in units of period 0 income, is denoted by $\pi_{s}$ for $s=1, \ldots, S$. Furthermore, $\pi_{0}=1$.

The agent can sell his endowment $\omega$, from which he can purchase on the contingent markets any consumption stream $c$ satisfying the budget inequality

$$
\sum_{s=0}^{S} \pi_{s} c_{s} \leqslant \sum_{s=0}^{S} \pi_{s} \omega_{s} .
$$

The agent's utility function and his endowment define an economy $\mathscr{E}=(u, \omega)$. The equilibrium state prices are determined by the notion of competitive equilibrium.

Definition 4.2. A competitive equilibrium for an economy $\mathscr{E}=(u, \omega)$ is a consumption vector $c^{*}=\left(c_{0}^{*}, \ldots, c_{S}^{*}\right)$ and state price vector $\pi^{*}=\left(\pi_{1}^{*}, \ldots, \pi_{S}^{*}\right)$ that satisfy the following conditions:

1. $c^{*} \in \arg \max _{c} u\left(c_{0}, \ldots, c_{S}\right)$ s.t. $\sum_{s=0}^{S} \pi_{s}^{*} c_{s} \leqslant \sum_{s=0}^{S} \pi_{s}^{*} \omega_{s}$,

2. $c^{*}=\omega$.

We present two sufficient conditions for the existence of an equilibrium with nonnegative state prices.

Proposition 4.3. Consider an economy $\mathscr{E}=(u, \omega)$. If the elementary utility function $v_{0}: \mathbb{R} \rightarrow \mathbb{R}$ is strictly monotonic and concave and $v: \mathbb{R} \rightarrow \mathbb{R}$ is monotone and concave, then a competitive equilibrium $\left(c^{*}, \pi^{*}\right)$ exists. Moreover, $\pi_{s}^{*} \geqslant 0$ for $s=1, \ldots, S$.

Let $\mathscr{U}$ denote the set of utility functions $u$ in which the elementary utility functions $v_{0}: \mathbb{R} \rightarrow \mathbb{R}$ and $v: \mathbb{R} \rightarrow \mathbb{R}$ are twice differentiable, $v_{0}^{\prime}>0, v_{0}^{\prime \prime} \leqslant 0$ and $v^{\prime} \geqslant 0, v^{\prime \prime} \leqslant 0$.

Proposition 4.4. Consider an economy $\mathscr{E}=(u, \omega)$. If $u \in \mathscr{U}$, then the competitive equilibrium $\left(c^{*}, \pi^{*}\right)$ is unique. Moreover,

$$
\pi_{s}^{*}=\frac{\frac{\partial u(\omega)}{\partial c_{s}}}{\frac{\partial u(\omega)}{\partial c_{0}}}=\frac{\bar{\delta} p_{s} v^{\prime}\left(\omega_{s}\right)}{v_{0}^{\prime}\left(\omega_{0}\right)} \geqslant 0, \quad s=1, \ldots, S
$$

The proofs of Propositions 4.3 and 4.4 are standard results in microeconomic theory, see for instance Mas-Colell et al. (1995).

Under the assumptions of Proposition 4.3 or Proposition 4.4 one can define the equilibrium price of any portfolio $Z \in \mathbb{R}^{S}$.

Definition 4.5. If $\left(c^{*}, \pi^{*}\right)$ is a competitive equilibrium of an economy $\mathscr{E}=(u, \omega)$, then the equilibrium price, $q_{Z}^{*}$ of the portfolio $Z \in \mathbb{R}^{S}$ is given by $q_{Z}^{*}=\sum_{s=1}^{S} \pi_{s}^{*} Z_{s}$. 
Note that under the assumption of Proposition 4.4 the equilibrium price of the portfolio $Z \in \mathbb{R}^{S}$ is uniquely given by

$$
q_{Z}^{*}=\sum_{s=1}^{S} \pi_{s}^{*} Z_{s}=\bar{\delta} \frac{\sum_{s=1}^{S} p_{s} v^{\prime}\left(\omega_{s}\right) Z_{s}}{v_{0}^{\prime}\left(\omega_{0}\right)} .
$$

As an important special case of Eq. (12), the discount factor becomes

$$
\delta=q_{\mathbf{1}}^{*}=\sum_{s=1}^{S} \pi_{s}^{*} 1=\bar{\delta} \frac{\sum_{s=1}^{S} p_{s} v^{\prime}\left(\omega_{s}\right)}{v_{0}^{\prime}\left(\omega_{0}\right)} .
$$

Note that the discount factor is endogenously determined in the exchange economy model.

\section{General equilibrium measures of risk}

Now we can define the GE measure of risk of a portfolio as the negative of its equilibrium market price.

Definition 5.1. Consider an economy $\mathscr{E}=(u, \omega)$ with competitive equilibrium $\left(c^{*}, \pi^{*}\right)$ and a domain $V$. The GE measure of risk is the map $\rho_{u, \omega}: V \rightarrow \mathbb{R}$, where $\rho_{u, \omega}(Z)=-q_{Z}^{*}$ for any $Z \in V$.

Note that Definitions 4.5 and 5.1 imply that for any $Z \in V$

$$
\rho_{u, \omega}(Z)=-q_{Z}^{*}=-\sum_{s=1}^{S} \pi_{s}^{*} Z_{s}
$$

Notice that $\rho_{u, \omega}$ is a linear function.

Example 5.2. Consider an economy $\mathscr{E}=(u, \omega)$ and a domain $V$. Let $v_{0}\left(c_{0}\right)=c_{0}-\frac{1}{2} \alpha c_{0}^{2}$ and $v\left(c_{s}\right)=c_{s}-\frac{1}{2} \alpha c_{s}^{2}$ for $s=1, \ldots, S$, where $\alpha \in \mathbb{R}_{++}$such that $1-\alpha \omega_{s}>0, s=0, \ldots, S$, i.e. the elementary utility functions are quadratic and increasing at $c^{*}=\omega$. Since $u \in \mathscr{U}$, using Eqs. (11) and (14) the GE measure of risk of $Z \in V$ is given by

$$
\rho_{u, \omega}(Z)=-q_{Z}^{*}=-\sum_{s=1}^{S} \pi_{s}^{*} Z_{s}=-\bar{\delta} \frac{\sum_{s=1}^{S} p_{s} v^{\prime}\left(\omega_{s}\right) Z_{s}}{v_{0}^{\prime}\left(\omega_{0}\right)}=-\bar{\delta} \frac{\sum_{s=1}^{S} p_{s}\left(1-\alpha \omega_{s}\right) Z_{s}}{\left(1-\alpha \omega_{0}\right)} .
$$

One can also look at the stochastic part of the aggregate endowment $\tilde{\omega}$ as representing the market portfolio, since it captures the aggregate uncertainties. Let us denote the total return of any portfolio $Z$ by $r_{Z}$, where the total return is the payoff vector divided by the price: $r_{Z}=Z / q_{Z}^{*}$. It is well known (see for instance Geanakoplos and Shubik, 1990) that with a quadratic utility function the CAPM formula holds. It relates the total return of any portfolio to the total return of the market portfolio as follows:

$$
E\left(r_{Z}\right)=r_{\mathbf{1}}+\beta_{Z}\left(E\left(r_{\tilde{\omega}}\right)-r_{\mathbf{1}}\right), \quad \text { where } \beta_{Z}=\frac{\operatorname{Cov}\left(r_{Z}, r_{\tilde{\omega}}\right)}{\operatorname{Var}\left(r_{\tilde{\omega}}\right)} .
$$

Note that the total return of the reference instrument is given by $r_{1}=1 / \delta$. From $q_{Z}^{*}=E(Z) / E\left(r_{Z}\right)$ and Eq. (16) it follows that

$$
\rho_{u, \omega}(Z)=-q_{Z}^{*}=-\frac{E(Z)}{r_{\mathbf{1}}+\beta_{Z}\left(E\left(r_{\tilde{\omega}}\right)-r_{\mathbf{1}}\right)} .
$$


Thus if the elementary utility functions are quadratic, then the GE measure of risk of portfolio $Z$ is its discounted expected loss $-E(Z) / r_{\mathbf{1}}=-\delta E(Z)$, corrected with its risk relation to the aggregate endowment.

Using the differentiability assumption let us denote the set of GE measures of risk on $V$ by $G_{V}$, where

$$
G_{V}=\left\{\rho_{u, \omega}: V \rightarrow \mathbb{R} \mid u \in \mathscr{U}, \omega \in \mathbb{R}^{S+1}\right\} .
$$

Let us discuss some properties of $G_{V}$.

Proposition 5.3. Any $\rho_{u, \omega} \in G_{V}$ is a coherent measure of risk.

Proof. By Proposition $4.4, \pi_{s}^{*} \geqslant 0$ for $s=1, \ldots, S$. Using this fact and the linearity of $\rho_{u, \omega}$ monotonicity, subadditivity and positive homogeneity follows immediately. For translation invariance note that $\delta=\sum_{s=1}^{S} \pi_{s}^{*}$ by Eq. (13). Then for all $\mathrm{Z} \in V$, for all $a \in \mathbb{R}$ such that $Z+a \mathbf{1} \in V$, we have

$$
\begin{aligned}
\rho_{u, \omega}(Z+\mathbf{1} a) & =-q_{Z+\mathbf{1} a}^{*}=-\sum_{s=1}^{S} \pi_{s}^{*}\left(Z_{s}+a\right)=-\sum_{s=1}^{S} \pi_{s}^{*}\left(Z_{s}\right)-\sum_{s=1}^{S} \pi_{s}^{*} a \\
& =-q_{Z}^{*}-\delta a=\rho_{u, \omega}(Z)-\delta a .
\end{aligned}
$$

Note that GE measures of risk are coherent measures of risk under the assumptions of Proposition 4.3 as well. Interestingly, in Proposition 6, Jaschke and Küchler (2001) presents a similar result for a family of normalized, nonnegative price systems.

As GE measures of risk are coherent and their linearity also implies comonotonic additivity, so far five out of six axioms of spectral measures of risk are satisfied by them. The sixth one is law invariance. The following example shows that GE measures of risk are not law invariant.

Example 5.4. As a special case of Example 5.2 consider 2 states of nature at $t=1$ with equal probability of occurrence. Let $\bar{\delta}=1$ and $\alpha=1$. Two portfolios, $X^{1}$ and $X^{2}$, the aggregate endowment, and the state prices calculated by Eq. (15) are given in Table 3.

Let us assume that $X^{1}, X^{2} \in V$. It is easy to see that $\widehat{X}^{1}$ and $\widehat{X}^{2}$ have the same probability distribution. However, $\rho_{u, \omega}\left(X^{1}\right)=0.9 \neq 1.05=\rho_{u, \omega}\left(X^{2}\right)$, so law invariance fails.

Note that the two portfolios in Table 3 are related differently to the economy. Portfolio $X^{1}$ is larger when $\omega$ is smaller, whereas $X^{2}$ is smaller when $\omega$ is smaller. Although the two portfolios have the same mean, $X^{1}$ is a better hedge against the aggregate uncertainty. That is why the GE measure of risk of $X^{1}$ is smaller than the GE measure of risk of $X^{2}$.

Table 3

A GE measure of risk which is not law invariant

\begin{tabular}{llllll}
\hline$s$ & $p_{s}$ & $X^{1}$ & $X^{2}$ & $\omega$ & $\pi_{s}^{*}$ \\
\hline 0 & - & - & - & 0 & 1 \\
1 & 0.5 & -1 & -2 & 0.2 & 0.4 \\
2 & 0.5 & -2 & -1 & 0.5 & 0.25 \\
\hline
\end{tabular}


Table 4

A GE measure of risk and the CAPM formula

\begin{tabular}{lcc}
\hline & $X^{1}$ & $X^{2}$ \\
\hline$E\left(X^{i}\right)$ & -1.5 & -1.5 \\
$\beta_{X^{i}}\left(X^{i}\right)$ & 0.7593 & -0.6508 \\
$\rho_{u, \omega}$ & 0.9 & 1.05 \\
\hline
\end{tabular}

One can easily calculate that in Example $5.4 r_{\mathbf{1}}=1.5385$, and $E\left(r_{\tilde{\omega}}\right)=1.7073$. Using those numbers we can apply Eq. (17) and calculate the GE measures of risk of $X^{1}$ and $X^{2}$ through the CAPM formula as it is given in Table 4. Note that $\beta_{X^{1}}$ is positive, whereas $\beta_{X^{2}}$ is negative.

Most of the GE measures of risk are not law invariant. In Example 5.4 all that is needed is that the state prices are different, which is the case in a generic economy. The failure of law invariance tells us that the stochastic part of the aggregate endowment (or market portfolio, in terms of CAPM) should be taken into account, when calculating the risk of a portfolio. Nevertheless one can generalize law invariance in such a way that GE measures of risk satisfy them. The point is to look at the joint distribution of a portfolio and the stochastic part of the aggregate endowment.

Definition 5.5. A measure of risk $\rho: V \rightarrow \mathbb{R}$ is generalized law invariant if for all $X, Y \in V$ such that for all $s=1, \ldots, S$

$$
\operatorname{Pr}\left(\widehat{X}=X_{s} \text { and } \hat{\omega}=\omega_{s}\right)=\operatorname{Pr}\left(\widehat{Y}=X_{s} \text { and } \hat{\omega}=\omega_{s}\right),
$$

we have $\rho(X)=\rho(Y)$.

Proposition 5.6. GE measures of risk are generalized law invariant.

Proof. It is easy to see from Eq. (12) and Definition 5.1 that any $\rho_{u, \omega} \in G_{V}$ is generalized law invariant.

\section{Connections: Spectral and GE measures of risk}

In this section we show the conditions under which spectral measures of risk as defined in Definition 3.1 are GE measures of risk as defined in Definition 5.1. Then we characterize GE measures of risk.

Definition 6.1. A vector $\tilde{\omega} \in \mathbb{R}^{S}$ and a set $V \subseteq \mathbb{R}^{S}$ are comonotonic if $\tilde{\omega}$ and $Z$ are comonotonic for every $Z \in V$.

Proposition 6.2 says that all the spectral measures of risk are GE measures of risk if and only if their domain is comonotonic with the stochastic part of the aggregate endowment, i.e. all the vectors in $V$ are comonotonic with $\tilde{\omega}$, their values go up and down together event by event.

Proposition 6.2. Assume $\omega$ is given and it has different outcomes in states $s=1, \ldots, S$. Moreover, let $V$ contain $S$ linearly independent vectors which are comonotonic with $\tilde{\omega}$. Then for every $M_{\phi}$ there exists a GE measure of risk $\rho_{u, \omega} \in G_{V}$ such that 


$$
M_{\phi}(Z)=\rho_{u, \omega}(Z) \quad \text { for all } Z \in V
$$

if and only if $\tilde{\omega}$ and $V$ are comonotonic.

Proof. Since $\tilde{\omega}$ has different outcomes in each state of nature by assumption, without loss of generality we can assume that $\omega_{1}<\omega_{2}<\ldots<\omega_{S}$.

$(\Leftarrow)$ We show that comonotonicity is sufficient. Take any spectral measure of risk, $M_{\phi}$ and any $Z \in V$. Since $\tilde{\omega}$ and $V$ are comonotonic, $\tilde{\omega}$ and $Z$ are also comonotonic. Let us search for a GE measure of risk $\rho_{u, \omega} \in G_{V}$ satisfying the equation $M_{\phi}(Z)=\rho_{u, \omega}(Z)$. Since $\omega$ is given, the only freedom is in choosing $u \in \mathscr{U}$. Using the definitions the requirement is

$$
M_{\phi}(Z)=-\delta \sum_{s=1}^{S} \phi_{s} Z_{s: S}=-\bar{\delta} \sum_{s=1}^{S} \frac{p_{s} v^{\prime}\left(\omega_{s}\right) Z_{s}}{v_{0}^{\prime}\left(\omega_{0}\right)}=\rho_{u, \omega}(Z) .
$$

From Eq. (13) the discount factor is determined as

$$
\delta=\bar{\delta} \frac{\sum_{s=1}^{S} p_{s} v^{\prime}\left(\omega_{s}\right)}{v_{0}^{\prime}\left(\omega_{0}\right)} .
$$

Using Eq. (19) and the assumption that $p_{1}=\cdots=p_{S}=\frac{1}{S}$, Eq. (18) reads as

$$
-\bar{\delta} \frac{\sum_{s=1}^{S} \frac{1}{S} v^{\prime}\left(\omega_{s}\right)}{v_{0}^{\prime}\left(\omega_{0}\right)} \sum_{s=1}^{S} \phi_{s} Z_{s: S}=-\bar{\delta} \sum_{s=1}^{S} \frac{\frac{1}{S} v^{\prime}\left(\omega_{s}\right) Z_{s}}{v_{0}^{\prime}\left(\omega_{0}\right)} .
$$

After simplifying and rearranging, Eq. (20) leads to

$$
\sum_{s=1}^{S} \phi_{s} Z_{s: S}=\frac{\sum_{s=1}^{S} v^{\prime}\left(\omega_{s}\right) Z_{s}}{\sum_{s=1}^{S} v^{\prime}\left(\omega_{s}\right)}
$$

In Eq. (21) two weighted averages of $Z$ should coincide. Since $\tilde{\omega}$ and $Z$ are comonotonic, a larger $\tilde{\omega}$ implies a larger or equal $Z$. Thus the ordered and the non-ordered statistics of $Z$ coincide, i.e. $Z_{s: S}=Z_{s}$ for all $s=1, \ldots, S$. The weights on the left-hand side are by definition normalized, nonnegative, and non-increasing. The weights on the right-hand side are also normalized, and it is easy to see that $v$ can be chosen monotone and concave to obtain the same weights.

$(\Rightarrow)$ We show that comonotonicity is necessary.

By assumption $V$ contains $S$ linearly independent vectors which are comonotonic with $\tilde{\omega}$. Let us denote them by $Z^{1}, \ldots, Z^{\bar{S}} \in V$, where $\bar{S}=S$. Since for all $\bar{s}=1, \ldots, \bar{S}$ we have that $Z^{\bar{s}}$ and $\tilde{\omega}$ and comonotonic, we have that

$$
Z_{s: S}^{\bar{s}}=Z_{s}^{\bar{s}} \text { for all } s=1, \ldots, S,
$$

that is the ordered and the non-ordered statistics of $Z^{\bar{s}}$ coincide. Since the proposition should hold for any given $M_{\phi}$, let us take a spectral measure of risk with different $\phi_{s}$ in each state of nature. We show indirectly that if $\tilde{\omega}$ and $V$ are not comonotonic, then there is no GE measure of risk $\rho_{u, \omega} \in G_{V}$ such that $M_{\phi}(Z)$ yields the same number as $\rho_{u, \omega}(Z)$ for all $Z \in V$.

If $M_{\phi}\left(Z^{\bar{s}}\right)$ yields the same number as $\rho_{u, \omega}\left(Z^{\bar{s}}\right)$ for $\bar{s}=1, \ldots, \bar{S}$, then using the logic of Eq. (21) and the result in Eq. (22) the following equations are satisfied:

$$
\sum_{s=1}^{S} \phi_{s} Z_{s}^{\bar{s}}=\frac{\sum_{s=1}^{S} v^{\prime}\left(\omega_{s}\right) Z_{s}^{\bar{s}}}{\sum_{s=1}^{S} v^{\prime}\left(\omega_{s}\right)} \quad \text { for } \bar{s}=1, \ldots, \bar{S}
$$


As the equations in (23) are homogeneous, we can assume that $\sum_{s=1}^{S} v^{\prime}\left(\omega_{s}\right)=1$. Since the vectors $Z^{1}, \ldots, Z^{\bar{S}}$ are linearly independent, the equations in (23) determine that

$$
v^{\prime}\left(\omega_{s}\right)=\phi_{s} \text { for } s=1, \ldots, S .
$$

Now take any $Z \in V$ which is not comonotonic with $\tilde{\omega}$ (such $Z$ exists since $\tilde{\omega}$ and $V$ are not comonotonic). It follows from the definition of comonotonicity that there exist states of nature $s_{1}$ and $s_{2}$ in which

$$
\omega_{s_{1}}<\omega_{s_{2}} \text { but } Z_{s_{1}}>Z_{s_{2}} \text {. }
$$

Using Eqs. (21) and (24) we get that $M_{\phi}(Z)=\rho_{u, \omega}(Z)$ if and only if

$$
\sum_{s=1}^{S} \phi_{s} Z_{s: S}=\sum_{s=1}^{S} \phi_{s} Z_{s} .
$$

Both sides of Eq. (26) are weighted averages of $Z$. The left-hand side assigns strictly decreasing weights to the ordered statistics of $Z$. The right-hand side assigns the same weights but due to the observation in (25) at least one higher outcome of $Z$ gets a higher weight, leading to

$$
\sum_{s=1}^{S} \phi_{s} Z_{s: S}<\sum_{s=1}^{S} \phi_{s} Z_{s} .
$$

We have a contradiction.

Note that we had to assume in Proposition 6.2 that $\tilde{\omega}$ has different outcomes in each state of nature. In a generic economy this assumption is satisfied. The second assumption was that $V$ contains $S$ linearly independent vectors which are comonotonic with $\tilde{\omega}$. It is easy to check that this assumption is satisfied if $V$ contains all the vectors which are comonotonic with $\tilde{\omega}$ or if $V$ contains a convex cone generated by $\tilde{\omega}$ and $S-1$ linearly independent vectors. However, if we only require that $V$ contains $\tilde{\omega}$, then Proposition 6.2 cannot be proven as the following example shows.

Example 6.3. Suppose $V=\{\tilde{\omega}, Z\}$, where $\tilde{\omega}$ and $Z$ are given in Table 5 .

Note that the vector $\tilde{\omega}$ and $V$ are not comonotonic in Table 5, since $Z \in V$ is not comonotonic with $\tilde{\omega}$. We show that in this example for every $M_{\phi}$ there exists a GE measure of risk $\rho_{u, \omega} \in G_{V}$ such that $M_{\phi}(Z)=\rho_{u, \omega}(Z)$ for all $Z \in V$, although $\tilde{\omega}$ and $V$ are not comonotonic. Using the normalized version of Eq. (21) we have that $M_{\phi}$ and $\rho_{u, \omega}$ lead to the same number for $\tilde{\omega}$ if

$$
\begin{aligned}
& \phi_{1}+2 \phi_{2}+3 \phi_{3}=v^{\prime}\left(\tilde{\omega}_{1}\right)+2 v^{\prime}\left(\tilde{\omega}_{2}\right)+3 v^{\prime}\left(\tilde{\omega}_{3}\right), \text { and for } Z \text { if } \\
& -2 \phi_{1}-1.25 \phi_{2}-\phi_{3}=-2 v^{\prime}\left(\tilde{\omega}_{1}\right)-v^{\prime}\left(\tilde{\omega}_{2}\right)-1.25 v^{\prime}\left(\tilde{\omega}_{3}\right) .
\end{aligned}
$$

Table 5

$Z$ is not comonotonic with $\tilde{\omega}$

\begin{tabular}{lll}
\hline$s$ & $\tilde{\omega}_{s}$ & $Z_{s}$ \\
\hline 1 & 1 & -2 \\
2 & 2 & -1 \\
3 & 3 & -1.25 \\
\hline
\end{tabular}


Moreover, we know that

$$
\begin{aligned}
& \phi_{1}+\phi_{2}+\phi_{3}=v^{\prime}\left(\tilde{\omega}_{1}\right)+v^{\prime}\left(\tilde{\omega}_{2}\right)+v^{\prime}\left(\tilde{\omega}_{3}\right)=1, \text { and by definition } \\
& \phi_{1} \geqslant \phi_{2} \geqslant \phi_{3} .
\end{aligned}
$$

Solving Eqs. (28)-(30) leads to the unique solution

$$
\begin{aligned}
& v^{\prime}\left(\tilde{\omega}_{1}\right)=\phi_{1}+\frac{1}{5} \phi_{2}-\frac{1}{5} \phi_{3}, \\
& v^{\prime}\left(\tilde{\omega}_{2}\right)=\frac{3}{5} \phi_{2}+\frac{2}{5} \phi_{3}, \\
& v^{\prime}\left(\tilde{\omega}_{3}\right)=\frac{1}{5} \phi_{2}+\frac{4}{5} \phi_{3} .
\end{aligned}
$$

Since $v$ is assumed to be concave,

$$
v^{\prime}\left(\tilde{\omega}_{1}\right) \geqslant v^{\prime}\left(\tilde{\omega}_{2}\right) \geqslant v^{\prime}\left(\tilde{\omega}_{3}\right) \text { should hold. }
$$

From Eq. (31) we have that $\phi_{2} \geqslant \phi_{3}$. This observation implies with Eq. (32) that $v^{\prime}\left(\tilde{\omega}_{1}\right) \geqslant \phi_{1}$; with Eqs. (33) and (34) that $v^{\prime}\left(\tilde{\omega}_{2}\right) \geqslant v^{\prime}\left(\tilde{\omega}_{3}\right)$; and with Eq. (33) that $v^{\prime}\left(\tilde{\omega}_{2}\right) \leqslant \phi_{2}$. Since $\phi_{1} \geqslant \phi_{2}$ from Eq. (31) the inequalities in Eq. (35) are clearly satisfied.

The strong requirement in Proposition 6.2 is that $\tilde{\omega}$ and $V$ should be comonotonic to represent all the spectral measures of risk by GE measures of risk. In this case all the vectors in $V$ are dependent in the strongest form on the stochastic part of the aggregate endowment, their value should always go up and down together event by event. The larger the number of states $S$, the more stringent this assumption is.

We can characterize the set of GE measures of risk as follows.

Definition 6.4. A measure of risk $\rho: V \rightarrow \mathbb{R}$ satisfies the pricing kernel property on $V$ if there exists a vector $\gamma \in \mathbb{R}_{+}^{S}$ such that for all $Z \in V$ we have that

$$
\rho(Z)=-\sum_{s=1}^{S} p_{s} \gamma_{s} Z_{s} .
$$

The vector $\gamma$ is interpreted as the pricing kernel, and the measure of risk of portfolio $Z$ is the negative of its price, induced by the pricing kernel. Let us denote by $P_{V}$ the set of risk measures satisfying the pricing kernel property on $V$. Then we have the following proposition.

Proposition 6.5. The set of GE measures of risk on $V$ coincides with the set of risk measures satisfying the pricing kernel property on $V$, thus $G_{V}=P_{V}$.

\section{Proof}

$G_{V} \subseteq P_{V}$. For any $\rho_{u, \omega} \in G_{V}$, by Eq. (14) we have that

$$
\rho_{u, \omega}(Z)=-\sum_{s=1}^{S} \pi_{s}^{*} Z_{s} .
$$


By Proposition $4.4, \pi^{*} \in \mathbb{R}_{+}^{S}$. Using the notation $\gamma_{s}=\pi_{s}^{*} / p_{s}, s=1, \ldots, S$, Eq. (37) can be rewritten as

$$
\rho_{u, \omega}(Z)=-\sum_{s=1}^{S} p_{s} \gamma_{s} Z_{s}, \quad \gamma \in \mathbb{R}_{+}^{S},
$$

thus $\rho_{u, \omega}$ satisfies the pricing kernel property.

$P_{V} \subseteq G_{V}$. Let us take any measure of risk $\bar{\rho} \in P_{V}$ satisfying the pricing kernel property on $\bar{V}$. By definition there exists a vector $\gamma \in \mathbb{R}_{+}^{S}$ such that for all $Z \in V$ we have that

$$
\bar{\rho}(Z)=-\sum_{s=1}^{S} p_{s} \gamma_{s} Z_{s} .
$$

We show that there exist a quadratic utility function $u$ and endowment $\omega \in \mathbb{R}^{S+1}$ such that for all $Z \in V$ we have that $\bar{\rho}(Z)=\rho_{u, \omega}(Z)$. Let $v_{0}\left(c_{0}\right)=c_{0}-\frac{1}{2} \alpha c_{0}^{2}$ and $v\left(c_{s}\right)=c_{s}-\frac{1}{2} \alpha c_{s}^{2}$ for $s=1, \ldots, S$. In equilibrium $c^{*}=\omega$. We know from Eq. (15) that

$$
\rho_{u, \omega}(Z)=-\bar{\delta} \frac{\sum_{s=1}^{S} p_{s} v^{\prime}\left(\omega_{s}\right) Z_{s}}{v_{0}^{\prime}\left(\omega_{0}\right)}=-\bar{\delta} \frac{\sum_{s=1}^{S} p_{s}\left(1-\alpha \omega_{s}\right) Z_{s}}{\left(1-\alpha \omega_{0}\right)} .
$$

Let $\alpha=1, \bar{\delta}=1$ and $\omega_{0}=0$. Then Eq. (40) simplifies to

$$
\rho_{u, \omega}(Z)=-\sum_{s=1}^{S} p_{s}\left(1-\omega_{s}\right) Z_{s}
$$

By setting $\omega_{s}$ such that $\gamma_{s}=\left(1-\omega_{s}\right), s=1, \ldots, S$, Eqs. (39) and (41) define the same measure of risk. As $v_{0}^{\prime}=1$ and $v_{s}^{\prime}=\gamma_{s}, s=1, \ldots, S$, the monotonicity requirements of the elementary utility functions are satisfied.

Thus GE measures of risk are the only measures of risk satisfying the pricing kernel property. They are linear functions induced by $S$ nonnegative parameters $\left(\gamma_{1}, \ldots, \gamma_{S}\right)$. On the other hand spectral measures of risk are piecewise linear functions, also induced by $S$ parameters $\left(\phi_{1}, \ldots, \phi_{S}\right)$, which are applied to the ordered statistics of the portfolio's payoff vector. Thus, although the cardinalities of their sets are the same, under some technical assumptions spectral measures of risk are GE measures of risk if and only if the orderings of all the portfolio vectors are the same as the ordering of the market portfolio.

\section{Conclusion}

In this paper we discussed coherent and spectral measures of risk from a general equilibrium (GE) perspective. Coherent measures of risk can be defined by four axioms: monotonicity, subadditivity, positive homogeneity, translation invariance. Adding two more axioms, law invariance and comonotonic additivity leads to spectral measures of risk. We considered the discrete setting and a general domain $V \subseteq \mathbb{R}^{S}$. We proved that it is also true in the discrete setting with unrestricted domain, i.e. if $V=\mathbb{R}^{S}$ that spectral measures of risk are the only coherent measures of risk satisfying law invariance and comonotonic additivity. However, we have shown examples where on a general domain $V$ this may not hold.

We defined a natural measure of risk coming out of a general equilibrium model. The GE measure of risk of a portfolio is the negative of its equilibrium market price. Checking 
the properties of GE measures of risk enabled us to assess the above mentioned six axioms. We found that GE measures of risk are coherent measures of risk. This way the four axioms of coherent measures of risk are supported from a general equilibrium perspective. Thus Value at Risk and other non-coherent risk measures cannot be associated with our general equilibrium framework.

However, GE measures of risk do not satisfy law invariance, but only a generalized version of it, in which the market portfolio is also taken into account. Since spectral measures of risk are law invariant, we can conclude that in general, when calculating the risk of a regulated entity, spectral measures of risk do not take into account its relation to the market portfolio, leading to an under- or overestimation of risk. The same idea is shown by our result that spectral measures of risk are GE measures of risk if and only if all the regulated entities are comonotonic with the market portfolio, i.e. their values go up and down together event by event. Finally, we showed that GE measures of risk are the only measures of risk satisfying the pricing kernel property, which means that any nonnegative pricing kernel can induce them as the negative of the equilibrium market price.

\section{References}

Acerbi, C., 2002. Spectral measures of risk: A coherent representation of subjective risk aversion. Journal of Banking and Finance 26, 1505-1518.

Acerbi, C., 2004. Coherent representations of subjective risk aversion. In: Szegö, G. (Ed.), Risk Measures for the 21st Century. Wiley, New York, pp. 149-207.

Acerbi, C., Tasche, D., 2002. On the coherence of expected shortfall. Journal of Banking and Finance 26, 14871504.

Artzner, P., Delbaen, F., Eber, J.-M., Heath, D., 1999. Coherent measures of risk. Mathematical Finance 9 (3), 203-228.

Duffie, D., 2001. Dynamic Asset Pricing Theory, third ed. Princeton University Press, Princeton.

Embrechts, P., McNeil, A., Straumann, D., 2002. Correlation and dependence in risk management: Properties and pitfalls. In: Dempster, M.A.H. (Ed.), Risk Management: Value at Risk and Beyond. Cambridge University Press, Cambridge, pp. 176-223.

Föllmer, H., Schied, H., 2002. Stochastic Finance: An Introduction in Discrete Time. Walter de Gruyter, Berlin.

Geanakoplos, J., Shubik, M., 1990. The capital asset pricing model as a general equilibrium with incomplete markets. The Geneva Papers on Risk and Insurance 15, 55-72.

Ibragimov, R., 2005. Portfolio diversification and value at risk under thick-tailedness. Harvard Institute of Economic Research Discussion Paper 2086.

Jaschke, S., Küchler, U., 2001. Coherent risk measures and good-deal bounds. Finance and Stochastics 5, $181-200$.

Leroy, S., Werner, J., 2001. Principles of Financial Economics. Cambridge University Press, Cambridge.

Lintner, J., 1965. The valuation of risky assets and the selection of risky investments in stock portfolios and capital budgets. Review of Economics and Statistics 47, 13-37.

Magill, M., Quinzii, M., 1996. Theory of Incomplete Markets. MIT Press, Cambridge, Massachusetts.

Marshall, A., Olkin, I., 1979. Inequalities: Theory of Majorization and Its Applications. Academic Press, New York.

Mas-Colell, A., Whinston, M.D., Green, J.R., 1995. Microeconomic Theory. Oxford University Press, Oxford. Nelsen, R.B., 1999. An introduction to copulas. In: Lecture Notes in Statistics, 139. Springer-Verlag, New York.

Sharpe, W.F., 1964. Capital asset prices: A theory of market equilibrium under conditions of risk. Journal of Finance 19, 425-442.

Tasche, D., 2002. Expected shortfall and beyond. Journal of Banking and Finance 26, 1519-1533. 